\title{
Healing Traumatic Memories in Complex PTSD
}

\author{
Vito Zepinic \\ PsychClinic P/L, London, UK
}

Email address:

vito@psychclinic.net

\section{To cite this article:}

Vito Zepinic. Healing Traumatic Memories in Complex PTSD. Psychology and Behavioral Sciences. Vol. 7, No. 1, 2018, pp. $21-28$. doi: $10.11648 /$ j.pbs.20180701.15

Received: January 29, 2018; Accepted: February 5, 2018; Published: June 15, 2018

\begin{abstract}
In clinical practice healing traumatic memories is fundamental for the entire treatment of complex PTSD. Clinicians are united in opinion that PTSD becomes treatment resistant when the patients process traumatic memories partially or fragmented. We emphasized that traumatic memories in complex PTSD are made up of information imprinted during traumatic event and activation of one aspect of them facilitates retrieval of one part, but inhibits the retrieval of others. While working on traumatic memories, the clinician is faced with the patient's images often being reported as if the event is occurring again with olfactory and auditory intrusions, intense emotions, sensations, and maladaptive physical actions and behaviours. Vehement emotions - the intense arousal evoked in trauma - prevent adaptive information processing and impair efforts to formulate the traumatic event into explicit narrative. Therapy techniques presented in this paper are part of the Dynamic Therapy model [1] - trauma-centred and patient-oriented therapy designed for severe and complex stress-related disorders.
\end{abstract}

Keywords: Traumatic Memories, Complex PTSD, Re-Traumatisation, Inhibition, Retrieval

\section{Introduction}

Traumatic memories are an imprint of traumatic event in which the settings and measures are out of the individual's ability to control; they are qualitatively different from normal narrative memory and include sensory, affective, motor reliving experience, and behavioural re-enactments. In complex PTSD, traumatic memories are often provoked by negative, senseless, or repugnant unwanted intrusive thoughts that may take form of ideas, images, and urges which persist despite the patient's intention to control or neutralise them [1]. Intrusive memories could be so realistic and frequent that impose patient's obsession about their contents. They provoke catastrophic worries about the basis in real-life ('here-and-now') circumstances; their content is usually unrealistic but associated to the traumatic experience, making patient unable to realise their tendencies, and they might represent complete antithesis of one's personal standing.

We consider that the core in defining complex trauma (PTSD) is an understanding of its dissociation and traumatic memories. Further, we assume that traumatic memories should be seen as a construct of trauma survivor's life which is only partly assessable and it is also partly accessible to the consciousness at a given time [2]. The patient's traumatic memory system (emotions, cognition, and tendencies to respond) is fulfilled with stored unconscious and triggered traumatic materials. Consequently, many of the essential elements in therapy - transference, working through, and restructuring - involve working with traumatic memories.

Intrusive thoughts are triggered by stimuli in the environment, and they show an evidence to the trauma survivor that trauma may re-occur at any time [1] [3] [4] [5] [6]. Avoidance of such cues is very common as patient is not in power over the intrusive thoughts, to control them or decrease their consequences. Intrusive thoughts prevent patient from thinking positively or making analytic answers about relations between trigger and intrusive thoughts. Because of the repeated behaviour induced by intrusive thoughts, it is common that trauma survivor is misdiagnosed of having severe obsessions which, in fact, imply that only obsessional thoughts about trauma are present. However, it is just the type of safety behaviour (survival skills) used to neutralise or to ease an impact of the intrusive thoughts [7]. This highlights the importance of careful assessment of such phenomena in case of one's chronic traumatisation. The therapeutic aim should not be to eliminate intrusive thoughts but helping the patient to (1) consider thoughts as senseless mental noise rather than sufficient threatening, and (2) learn to manage acceptable levels of risk, uncertainty, and doubt 
associated with the intrusive thoughts [1] [8].

Intrusive thoughts imposed by any trigger are often accompanied by distressing physical response which seems like urges to act on violent (dangerous) circumstances, as it had happened during trauma experience. This makes patient's reactions similar to what happened during trauma when survival skills had been a habitual response to the real danger [7]. For example, one of our patients who had been tortured during war-imprisonment would always run into the corner of a room at any sudden sound of door opening. He explained being totally unaware of his reaction, but sudden sound was a trigger reminding him of his interrogator coming into the prison cell. He interpreted his escalating feelings of anxious arousal as an indication that he will be tortured again, and arousal was often accompanied by somatic reactions (sweating, trembling, or uncontrollable passing urine) with blackout of his mind, unable to think anything else but to 'prepare' his body and mind for torture.

Evoked by the trigger, the traumatic memories represent an intrusive return of unassimilated (non-coded) material in fragmented, sensory, affective, and motoric form. We emphasised four features that distinguish traumatic memories from the normal narrative memories [1]:

1. Traumatic memories are composed of the images, sensations and affective states; narrative memories are semantic and symbolic,

2. Traumatic memories are inflexible and unstable (invariant) over time; narrative memories serve one's social and adaptive function,

3. Traumatic memories cannot be evoked but elicited under specific circumstances (triggers) of the original event (s); narrative memorise are assessible without trigger, and

4. Traumatic memories take time to be remembered; narrative memories are common response on social demands.

\section{Treating Traumatic Memories}

Before commencing any therapy on traumatic memories, the clinician should be aware of patient's fear to release them. Many clinicians [1] [3] [4] [9] [10] [11] [12] are of opinion that the treatment work on traumatic memories is fundamental for the entire treatment of trauma-related problems. They are also of opinion that successful processing of the traumatic experience requires specific therapeutic efforts and that any failure to process traumatic memories inevitable leads to a chronic condition of PTSD. Some clinicians [13] proposed that PTSD becomes treatment resistant when the patients process traumatic memories in a way that retains a sense of persistent threat or danger. Recalling traumatic memories is often characterised by multiple repetitions, confusions and fragmentations making traumatic memories a unique clinical phenomenon.

Clinically, these fragmentary qualities are experienced as intense sensory components such as vivid visual images, sounds, feelings, or sensations associated with the traumatic experience. Further, individuals with complex PTSD often have difficulties in generating a coherent verbal narrative of the traumatic event; instead, the narrative is characterised by frequent repetitions, specific fillers, and temporal sequencing problems [14].

Unlike in simple PTSD, the process of desensitisation (freedom from the fears of a danger) in complex trauma requires different techniques with ruled out in vivo exposure. In complex PTSD, vivid memories and maladaptive behaviour generate havoc on the patient's functioning. Vehement emotions - the intense arousal evoked in trauma prevent the adaptive information processing and impair efforts to formulate traumatic memories into explicit narrative. The unconscious nature of the traumatic memories is efficient; they give rise to all maladaptive emotions, thoughts, sensations, perceptions, and behaviours. As the work on memories commences, the experience of high arousal is a common experience during therapy. As the inner conflict drives are still powerful, the patient is reluctant to release traumatic memories; even exists a fear of retraumatisation because of retrieval of the traumatic memories. However, through emphasis on the resolution and prevention of bottom-up hijacking by attending to the somatic sensations and movements, rather than to the emotional and cognitive dimensions, arousal is brought back into a window of tolerance [14].

Fear of re-traumatisation and return of the painful experience is based on fact that retrieval of traumatic memories requires [10]: (1) overcoming the phobia of the dissociative sense of self associated with the trauma, as well as fear and shame associated with thinking about the trauma; (2) overcoming phobia of the dissociated traumatic memories, which must be uncovered and transformed from the intrusive re-experiencing of the trauma-related feelings and sensations to a trauma-related narrative within the personal stream of consciousness, and (3) overcoming the phobia of life itself, which includes the fear of being revictimized and the feelings that the victim will be unable to take charge of his own destiny. The clinician should be certain about the patient's preparedness for the memory retrieval, otherwise condition could further deteriorate.

The main issue in working on traumatic memory retrieval is a secure bond between the clinician and the patient - the presence of an attachment figure which provides patient with the security necessary to explore his trauma experience and to interrupt the inner or social isolation that keeps patient stuck in the repetitive patterns [1] [11]. Treatment does not necessarily involve repeated recollection of traumatic memories, but assists patient in recognising the imprint of past trauma in current circumstances ('here-and-now'). Complex trauma (PTSD), usually caused by prolonged or repeated traumatic experience, cannot be conceived as unitary experiences, as the trauma itself, with its duration and combination of trauma experiences, is specific to the individual case. Not only are traumatic experiences different from case to case, but each traumatised individual internalises these experiences with own cognition and affects. 
Traumatic memory is both the precursor and the contributor to the personality pathology, and it is interpreted through the nature of patient's current comorbid personality organisation.

What is important in understanding traumatic memories is the manner of traumatic experience which had formed, remembered, and integrated memories into the personality structure. Using the Dynamic Therapy model [1] [11] [16] [17] in treating severely traumatised individuals, we treated traumatic memories in combining interplay techniques of: (1) imagery exposure, (2) cognitive reconstruction, (3) drawing or writing story, and (4) self-instructional training. Dynamic Therapy is three-phased patient-oriented model which applies to holotropic integration of the distorted self into a whole using three main concepts: (a) restoration of a form of the relatedness ('Interconnectivity'); (b) restoration of a sense of the aliveness/vitality ('Dynamism'); and (c) restoration of an awareness of self and inner events ('Insight').

\subsection{Imagery Exposure}

Considering that in vivo exposure is not applicable in treating complex PTSD traumatic memories, the imagery exposure is used in order to avoid disintegration of the traumatised personality or revictimization. The aim of imagery exposure is to help patient to confront fears and/or trauma-evoking stimuli and weaken distressing thoughts, emotions, and somatic arousal associated with the trauma through habituation and correction of catastrophic misinterpretation of the presence and significance of such experience. Before commencing treatment, the clinician should analyse patient's unconscious 'identification with trauma' in order to avoid: (1) displacement of the conflicts outside the transference; (2) the maintenance of the splitting of therapeutic relationship; and (3) continuation of the severe pathology and inhibition that can frequently evolve during 'recreative trauma' process [1] [11].

In the race to prevent revictimization, it is important that the patient is aware of these possible risks in therapy and be able to differentiate 'here-and-now' mode from the traumatic past ('then-and-there'). Coming to terms with the past involves recognition of the fears, pain, and terror, as well as rage or hatred derived from a painful invasion of personality boundaries and threatening effects. Facing traumatic past also involves recognising what intruded parts of the traumatic experience are the most powerful that might resist any changes in personality. Fixation on traumatic past will identify sequences of traumas and their violent destruction on the personality. This will also identify the efforts to transform traumatic painful experience into an adaptive part of the personality functioning.

Traumatic memories are complex and difficult material and many trauma survivors have significant gaps in their memories of trauma experience, or show dissociation. In clinical practice, it is quite difficult to reconstruct one's traumatic experience without causing re-traumatisation which can be within the original trauma, characterised by repeated traumatic episodes and their long-term aftermaths. Any risk of re-traumatisation and protective factors should be identified and then discussed with patient prior to starting therapy session of the imagery exposure. Treatment of the complex traumatic memories and their retrieval cannot be an unrealistic clinician's promise that 'therapy will work'; otherwise condition regression may occur with further encapsulation of the inner conflicts and unconscious memories [11] [18] [19]. The real task is to bring the patient face-to-face with horrible past and to integrate fragmented parts of the personality into a fully developed life narrative to overcome the terror of the traumatic event by exposing patient to a controlled relieving experience [1] [4].

Overcoming fears of releasing traumatic memories requires gradual and guided approach which is directed by the clinician and patient's awareness how memories impact on patient's personality. Thus, the principal elements of therapy are the transformation of traumatic memories into a symbolic verbal or visual account that is fully personified and presentified. This realisation results in an autobiographical narrative memory of traumatic event and in actions that can be adapted to the present rather than to the traumatic past [9].

The Imagery exposure therapy involves two major components: guided realisation and guided synthesis of traumatic memories [1] [11]. Guided synthesis involves modulated and controlled exposure to the traumatic memory in which the patient receives help from the clinician to remain oriented and focused on the present time while synthesising the previously dissociative mental content of the traumatic memories. In essence, these are the affective, cognitive, sensorimotor, and behavioural components of memory associated with various dissociative parts of personality. Guided realisation is an ongoing therapy process of helping patient to realise the background of losses and suffering, and to move forward towards the higher levels of (adaptive) emotions, thinking, and behaviours.

Imagery exposure is a systematic method to gradually 'expose' patient to traumatic moments and desensitise catastrophic reactions. This strategy allows patient to confront an otherwise covert event and manipulation of the 'here-and-now' situation by the traumatic past. Therapy is based on patient's repeatedly experiencing 'trauma exposure' in a safe and controlled manner by the clinician:

'I want you to close your eyes and imagine yourself in a garden of your home on a spring sunny day. You are sitting in a comfortable chair, drinking coffee, and watching a blossom in the neighbour's orchard. The sky is blue with a breeze and some clouds are floating above. You feel happy and relaxed. You smell the sweet smell of grass. Your interrogator is walking on the street with his head down avoiding eye contact with you. You can hear birds singing, short pure whistles like saying to your interrogator he is a bad person. You can almost imitate them. Looking closer, you see your interrogator's red sweaty face but you do not feel any hatred or hurt... you just enjoy the sunny day. You feel completely calm and safe - just enjoy this scene for a few minutes'.

In imagining these scene, the patient feels fully relaxed moving away his thinking about the interrogator and torture. 
Of course, the clinician expects a lot of the patient's startle responses (emotional and/or physical) while mentioning the interrogator and torture. It is also possible that patient stops imagery due to the extreme reactions and feelings, and clinician should encourage patient to disclose feelings and thoughts that stop imagery exposure. The imagery should be repeated as much as needed until the patient achieved relaxed and safe feelings and thinking about the trauma event even as if it has not occurred. Every therapy session should use different imagery scenes in order to avoid 'learned condition' that could make imagery be ineffective.

During the imagery, the patient substitutes unpleasant memory with a pleasant imagery which is non-anxiety triggering. The purpose of the imagery exposure is to achieve mastery over repetitive visual/olfactory/ auditory/sensory stimuli that causes fears of the traumatic memories. Also, being free of traumatic memories impact, inner impulses, negative thoughts, maladaptive emotions, and behaviour, the patient sufficiently increases sense of self and personality functioning [1] [4] [20] [21]. In order to reduce fears of the retrieved traumatic memories, the patient could be instructed to reveal memories as a 'third person', as if the memories and traumatic experience do not belong to the patient. This keeps the patient distant from the emotional experience and thoughts of the trauma. Some patients stated that, during this process, they felt as if they were watching themselves and do not feel to stressed about 'third' person's feelings or thoughts.

In many complex trauma cases, there are some 'hotspots' of traumatic memories which might be so extreme and horrific; they play a significant role in overall comorbid condition and patient is reluctant to retrieve them. The 'hotspots' often correspond to the elements of the traumatic memories that usually intrude during nightmares, unwanted memories, and flashbacks [22]. Before retrieval of 'hotspots', it is helpful to elaborate them as if happened to the 'third' person not to the patient. Careful preparation with guided approach maximises the probability that the patient's mental strength is high enough to take steps towards the therapy on own 'hotspots'.

\subsection{Cognitive Reconstruction}

The initial goals of cognitive reconstruction are development of self-awareness and helping patient to restructure his thinking by being aware of negative thought processes. Reconstructing thoughts is a necessary step in correcting distortions, and when increasing self-awareness, the patient realises how sufficient is to correct his errors in thinking. Self-awareness allows the patient to distant himself from faulty thinking and to develop a more objective perspective towards situation [23]. With reported negative thoughts by the patient, the clinician becomes more aware and understands the patient's vulnerability, weakness, and difficulties in controlling his emotions and thoughts associated with complex trauma syndrome [1]. Together with patient, the clinician, for example using a marker board, may summarise all negative thoughts and categorise them in an order of their meaning to the patient.

Frightening thoughts may cause other associated distortions such as emotions, behaviour, or relatedness, so that the patient should clarify which thoughts are the most frightening and/or disturbing, and should be processed in therapy first. Sometimes a visual reminder on the marker board triggers patient differently than was thought. Cognitive reconstruction is a therapy process which actually transforms the traumatic memory so that it can be integrated into the trauma survivor's present life without fears. The concept of thoughts reconstruction developed in the Dynamic Therapy model is based on Janet's description [24] of normal memory as 'the action of telling a story'. Although the traumatic memories are often deeply unconscious, the clinician overtakes role as a 'witness and ally' who encourages the traumatised individual to speak freely and openly about something that seems unspeakable. This reconstruction process demands courage and therapeutic alliance of both the clinician and the patient. The clinician needs to clarify the severity of the meaning of negative thoughts to the patient so that more meaningful ones start to reconstruct [1].

However, for the therapy process is important to grade how much thoughts caused fears and distortion of the patient's feelings and thinking. The patient usually withholds reporting the most frightening thoughts until the end due to cognitive avoidance. Most patients find that seeing their thoughts written on the marked board is less frightening than they expected and, thus, are stimulated to overcome their avoidance and search out other frightening thoughts. Because patients experience many of their fears in the forms of vivid pictures, they often respond well when their fears are illustrated with stick or symbolic drawings [23]. Rating the negative thoughts also helps patients to observe the own self and capacities to master the thoughts and associated problems.

After seeing his thoughts written on the marked board, the patient can eventually decide from which thoughts and behaviour he may distance himself. Some will, for the first time, become aware of real problems associated with negative thoughts which they have been previously scared to rate, analyse, or even think about. With a rating list, the clinician may, together with the patient, conceptualise the patient's problems and to plan the therapy strategy [1]. Alongside with writing on the marked board, it is useful technique of 'mirroring' to evaluate disturbing thoughts and feelings. The 'mirroring' is proto-conversation and primary intra-subjectivity that is attuned to the patient's present state. It is simple reflecting back of the traumatic experience of the 'other' [25]. The role of vision is interaction with the 'other', who is not able to respond or argue about the patient's narrative. The patient and the 'other' in the mirror have fixed eyes upon each other. It is a marching process, which is not equivalent to imitation as imitation is static with nothing moving beyond it. In other words, the 'mirroring' is a dynamism between the patient and the 'other'.

Matching responses in 'mirroring' are an essential element of the self-awareness and this fundamental interplay involves 
facial expression, but above all thoughts and narrative. The proto-conversation goes in a state of positive thoughts affect mediated by expression and vocalisation. The subject failure (traumatised self with maladaptive thoughts, emotions, and behaviour) is caught in a cage of the mirror [1]. The clinician instructs patient to express his thoughts and feelings towards the 'other' who cannot respond, making the patient's safety intact. Because of this, the patient intensely expresses his personal meaning as if passing to the 'other' not to himself. Vocalisation, facial expression, and body movements of the two 'partners' fit in a complex matching that leads to an escalation of the patient's thoughts, emotions, and their expression.

While implementing 'mirroring' we required traumatised individual to sit in front of the mirror and become aware of his thinking seeing 'other'. A mirror is a 'social window' or 'other person' that was being told about the patient's thoughts and feelings. Upon looking in the mirror, the patient talks to the 'other person' (which could also be imaginative) identifying the fear-produced thoughts that influence patient's maladaptive feelings, behaviour, and self-image. We realised how much the patient lacks in narrative while explaining to the 'other' the background and nature of thoughts distraction. The clinician suggests that patient's free-association of thoughts be reported and to evaluate level of meaningfulness to the patient [1].

It is particularly important to record free-associated thoughts and emotions of brutally raped patients, or those who have been exposed to prolonged or repeated violence and torture. The thoughts and feelings have often been addressed with fear and anger. We supposed that retrieval of traumatic memories could be effective if trauma survivor talks to the 'perpetrator or rapist in the mirror' with no fear of any consequences. This catharsis method has significantly helped in thoughts reconstruction but, more importantly, improved the patient's self-image and strength, and empowered patient to disclose his traumatic memories without fear of 'being punished'. The patient must feel powerful to direct his attention to the phenomenon that hurts the most and revokes the main aspects of his horrible experience. We also emphasised that, in complex trauma, there is a formation of massive and unconscious repression from the inner conflicts in which the traumatised individual become entirely 'frozen' and amnestic due to the repeated or prolonged trauma experience. We assume that broadly encompassing amnesia may be more likely to involve dissociative pathology than unconscious 'repression' by the inner conflict drives [11].

The main purpose of uncovering traumatic memories is to gain conscious control over unbidden re-experiences or reenactment of the trauma so that the patient becomes increasingly able to control the present thoughts and feelings. Some clinicians [19] [26] suggest that some patients with severe traumatic memories make conscious effort to suppress thoughts and memory of the horrible experience. However, this 'blocking it out' approach usually does not bring completely non-coded memories which potentially can be revived by a trigger even several years after the original trauma. This fact could make difficulties during therapy as patient may require 'Doctor, please do not refresh my mind to it'. The clinician is then in a position to design cognitive reconstruction with no re-victimisation. Traumatic memories are an invasive and painful comorbid condition that reminders associated with them could be quite dangerous.

Reconstruction of the traumatic memories is quite ambitious and demanding task, and intrusive symptoms must be monitored carefully so that therapy is within the realm of what is bearable. Reconstructed trauma experience is an 'unwelcome' reminder to the patient of his past that may create contemptuous, hateful, outraged, or resentful feelings. Some patient may 'chose' to recall and to reconstruct only fragmented traumatic memories as all material is too painful and scary.

\subsection{Drawing or Writing a Story}

Non-verbal communication can be very helpful in treating those patients who had been exposed to horrible terror in captivity. The visual nature of traumatic memories in drawings may represent the most descriptive approach to those 'indelible images' of horror [27]. It is important to instruct patient to report in the most descriptive way what he saw, smelt, heard or felt during the traumatic experience - the bodily sensations that are important in reconstructing traumatic memories [28]. The patient should be specifically asked to write about smells, racing of heart, muscle tension, weakness of some parts of the body, etc. what is much easier for the patient than to talk about bodily sensations. The trauma victims often try to divert themselves from the painful memories, thoughts, and feelings, and, consequently, they are unable to recognise what is causing their negative feelings and/or catastrophic thoughts [23].

The primary strategy in writing dysfunctional thoughts is to teach patient to recognise automatic thoughts that appear outside the therapy session and to strive more alternative views. In daily recoding form of dysfunctional thoughts, the patient report (1) situation leading to anxiety or fear, (2) emotions felt and degree of emotions, and (3) automatic thoughts and degree of belief in the thoughts. Daily recording is the patient's 'homework' and it is an integral part of the therapy; when the patient recognises automatic thoughts he also better understands their relations and negative impact on his entire psychological equilibrium. With the clinician's support, when the patient is able to master this skill, then he is ready to provide the rationale response upon dysfunctional thoughts. Recording dysfunctional thoughts may sometime be difficult, as the patient feels that in doing that he could become even more anxious and experience fears of traumatic memories and associated thoughts.

Drawing or writing about thoughts and emotions is much easier than narrative reporting. The phenomenon in 'translating' traumatic memories into words can be also complicated by the fact that severely traumatised individuals have rarely experienced a single episode of the trauma. Exposure to sustained, repeated, or multiple traumas has been 
found to result in a complex symptom presentation that includes not only chronic PTSD but also other symptomatology reflecting the disturbances predominantly in the affective and interpersonal self-regulatory capacities such as difficulties with anxious arousal, anger management, dissociative symptoms, and aggressive or socially avoidant behaviour [29]. Because of multifactorial symptoms, complex PTSD is often syndromal condition caused by cumulative traumatic memories [30].

\subsection{Self-Instructional Training}

The anxiety and fears eliciting self-statement and irrational beliefs of danger and threat are of the nature of automatic, overlearned, and one's clue-producing responses, and they are not easily modifiable. Promoting general selfunderstanding without full awareness of the inner conflicts may lead the patient to a phobic state that, in case of erupted impulses, he becomes unable to control his personality and behaviour. Thus, the clinician should employ the selfinstructional training procedure in order to cognitively restructure unrealistic anticipations of probable response outcomes. The training is based on an assumption that the inner conflict drives obey the same ways of imprint as the overt behaviours do, and can effectively extinguish phobic behaviour [1].

Self-instructional training involves the following steps [1]: (1) explaining that unrealistic fears and anxiety are the result of their distressing things one tells himself about situation rather than due to the situation itself; (2) training the patient to identify specific self-verbalisation which he emits in the anxiety and fears eliciting situation and of which the trauma victim has been unaware (unconscious); (3) convincing the patient of the irrational and self-defeating nature of the inappropriate anxiety and fears generating self-statement; and (4) modelling and reinforcing alternative, constructive selfverbalisation which are incompatible with the anxiety and fears. It must be emphasised that the training is not designed to 'give inside' suppressed unconscious conflicts but rather created to promote the patient's awareness of his verbal and non-verbal behaviour taking into account immediate functional relationship with the emotional responsiveness. Although having some concept of 'insight-oriented' attitudes, the training is more oriented to 'controlling variables' that cause an unrealistic anticipation.

The self-instructional training has been developed from the cognitive-rational-emotional approach with a basis that much, if not all, dysfunctional behaviour and emotions are due to the irrational ways in which trauma victim construes his world. In severely traumatised individuals there is recognisable presence of numerous fundamental and widespread irrational attitudes which require reshaping into the rational judgment before the patient can free himself of incapacitating emotional propositions. In essence, the selfinstructional training relies on a basic necessity that the humans need to be loved and approved by others in an environment where they belong.

The central aim of self-instructional training is to make trauma victim able to overcome fears of inner trauma-derived feelings, thoughts, sensations, and memories. The conflicts and cognitive distortions can be understood in terms of these fears and patient makes a range of irrational actions in order to avoid or escape from certain trauma-related inner impulses. We emphasised that fears and inner conflicts are based on suppressed perceptions, goals, values, cognitions, and physical sensations during trauma that should be released [1]. Because of the specific fears of traumatic memories and dissociative parts of the personality, the self-instructional training is slow process which should be supervised, otherwise, it may create serious patient's internal distresses and ambivalent behaviours. Training begins with the systematic evaluation of the patient's mental actions that are tolerated (adaptive) or avoided (maladaptive) and the specific factors that maintain fears, maladaptive beliefs, and affect dysregulations.

During patient's narrative retrieval of the traumatic memories, the clinician observes them and makes assumptions as to how big impact they have upon the patient's personality and functioning, and what meaning they have. However, sometimes the patient is unable to release traumatic memories or identify negative thoughts and emotions. This is in situation when he distances himself from his thoughts or has a false sense of mastery over negative thoughts and memory. The patient may also realise that some part of the traumatic memories 'missing in action' making narrative of the memories fragmented and inaccurate. The clinician should evaluate partially released memories of how much unreleased material is distressful to the patient and would release of that potentially cause re-traumatisation and/or re-victimisation. However, whatever looks dangerous for the patient in case of releasing 'hidden scars', unconscious memories cannot be left to stay 'frozen' deeply inside. The best support to work on deeply unconscious memories is patient's desire to release all memories and transform them from maladaptive power into patient's controlled emotions, thoughts, and behaviour.

\section{Conclusion}

Bringing a stored traumatic material into consciousness is an inevitable part in treating complex stress syndrome. In clinical practice, it is common that people with the experience of a chronic traumatisation do not respond to retrieval of traumatic memories in the way like other stressaffected people do; during therapy process they may feel or act as if they were being traumatised all over again. They may experience the fear reactions from the point at which the danger is first perceived as real and then to the point at which one's behaviour, affect, and cognition are totally disorganised [31]. The phenomena of generalisation and incubation still further emphasise the fear-reaching of the trauma with usually collapse of the whole organisation of behaviour. Thus, the high states of arousal seem to selectively promote retrieval of traumatic memories, sensory information, or behaviours associated with the condition prior traumatic 
experience [32].

Memories of the trauma (somatic or symbolic) are elicited by a high emotional arousal or altered state of mind in case when trauma victims are brought back to the particular state of mind that occurred during the trauma. Memory retrieval may be involved in dissociative phenomena in which the traumatised person may be wholly or partially amnesic for memories or behaviour appeared in altered states of mind. Evoking traumatic memories is a unique event which requires a highly structured procedure and the therapeutic strategies. Sensory-reached, fragmented or poorly elaborated memory of trauma cannot be accommodated with other autobiographical memories and maintain a low activation threshold. They provide recurring confirmatory evidence for the negative beliefs about the self, world, future, and trauma [33]. These negative core prejudice one's memory recall so that trauma victim recalls aspects of the trauma that are congruent with the dysfunctional trauma schemas [1]. Thus, as the traumatic memories are related to the one's dysfunctional schemas about self, world, and future, the aspects of trauma will be recalled to confirm existence of such negative beliefs. Such elaborated schemas into memory during the trauma are inaccessible for retrieval as they are not consistent with post-trauma schemas.

Recalling traumatic memories is a reconstructive, not a reproductive, process in which trauma victim is not replying traumatic past like a videotape. In fact, recollection is based on an evidence what the patient remembers, an event from the past reconstructing it from encoded elements in the brain. Due to the deeply unconscious and not autobiographical nature, recalling traumatic memories in complex trauma are often episodic or fragments instead of being entirely released. Even though, the recalling fragments do not often correspond to originality (severity and complexity) of the suffering. It could be just as vivid as the fragmented memory of the event can be recalled - repisodic memory constructed from some episodes but not whole traumatic event (s).

\section{References}

[1] Zepinic V.: Hidden Scars: Understanding and Treating Complex Trauma, Xlibris, London, 2011.

[2] Zepinic V.: Healing traumatic memories: A case study, Dinamische Psychiatrie, 5-6, 279-287, 2008.

[3] Courtois CA. \& Ford JD.: Treating complex traumatic stress disorder, The Guilford Press, New York, 2009.

[4] Herman J.: Trauma and recovery, Basic Books, New York, 1992.

[5] van der Hurt O. Steele K. Boon S. \& Brown P.: The treatment of traumatic memories: Synthesis, realisation, and integration, Dissociation, 6, 162-180, 1993.

[6] van der Kolk BA. \& Fisler R.: Dissociation and the fragmentary nature of traumatic memories: Overview and explanatory study, Journal of Traumatic Stress, 8, 607-627, 1995.
[7] Zepinic V.: Persistence of 'Survival Skills' as a Risk Factor for Suicide in Severely Traumatised Individuals, International Journal of Emergency Mental Health and Human Resilience, 2, 552-558, 2015.

[8] Ulman RB. \& Brothers D.: The Shattered Self: A Psychoanalytic Study of Trauma, The Analytic Press, Hillsdale, 1988.

[9] van der Hurt O. Nijenhuis E. \& Steel K.: The haunted self, WW Norton, New York, 2006.

[10] van der Kolk BA. MacFarlane A. \& Weiseath L.: Traumatic Stress, The Guilford Press, New York, 1996.

[11] Zepinic V.: Trauma-focused Dynamic Therapy model in treating complex psychological trauma, Psychology, 8, 20592101, 2017.

[12] Zlotnik C. Warshaw. Shea MT. Alsworth J. et al,: Chronicity in posttraumatic stress disorder (PTSD) and predictors of course of comorbid PTSD in patients with anxiety disorders, Journal of Traumatic Stress, 12, 89-100, 1996.

[13] Ehlers A. \& Clark DM.: A cognitive model of posttraumatic stress disorder, Behavioural Research and Therapy, 38, 319$345,2000$.

[14] Rosen GM.: Posttraumatic Stress Disorder: Issues and Controversies, Wiley \& Sons, Chichester, 2004.

[15] Ogden P. Minotn K. \& Pain C.: Trauma and the Body, WW Norton, New York, 2006.

[16] Zepinic V.: Treating the war-related complex trauma using Dynamic Therapy, The International Journal of Medicine, 3, 384-390, 2010.

[17] Zepinic V.: The Dynamic Therapy Model in Treating Complex Trauma Syndrome, Studies in Sociology of Science, 4, 1-18, 2016.

[18] Courtois CA.: Complex trauma, complex reactions: Assessment and treatment, Psychotherapy: Theory, Research, Practice, and Training, 41, 412-425, 2004.

[19] Foa EB. \& Rothbaum OB.: Treating the Trauma of Rape, The Guilford Press, New York, 1998.

[20] Friedman JM. Keane MT. \& Resick AP.: Handbook of PTSD, Science and Practice, The Guilford Press, New York, 2007.

[21] Wilson JP. (ed.):: The Posttraumatic Self, Routledge, New York, 2006.

[22] Holmes EA. Grey N. \& Young KAD.: Intrusive images and 'hotspots' of trauma memories in posttraumatic stress disorder: An explanatory investigation of emotions and cognitive themes, Journal of Behavioural Therapy and Experimental Psychiatry, 36, 3-17, 2005.

[23] Beck AT. Emery G. \& Greenberg RL.: Anxiety Disorders and Phobias: A Cognitive Perspective, Basic Books, New York, 2005

[24] Janet P.: The Psychological Evolution of Personality, Chachine, Paris, 1929.

[25] Meares R.: Intimacy \& Alienation, Routledge, London, 2000.

[26] Williams LM.: Recovered memories of abuse in women with documented child sexual victimisation histories, Journal of Traumatic Stress, 8, 649-673, 1995. 
[27] Danieli Y.: Psychotherapists' participation in the conspiracy of silence about the Holocaust, Psychoanalytic Psychology, 1, 23-42, 1984.

[28] Wilson JP. \& Keane TM.: Assessing Psychological Trauma and PTSD, The Guilford Press, New York, 1996.

[29] Cloitre M. Stolbach CB. Herman LJ. van der Kolk BA. et al.: A developmental approach to complex PTSD: Childhood and adult cumulative trauma as predictors of symptoms complexity, Journal of Traumatic Stress, 5, 399-408, 2009.

[30] Zepinic V.: The Self and Complex Trauma, Xlibris, London, 2012.
[31] Zepinic V.: Disintegration of the Self-Structure Caused by Severe Trauma, Psychology and Behavioural Science, 4, 8392, 2016.

[32] American Psychiatric Association.: Diagnostic and Statistical Manual of Mental Disorders (DSM-5), APA, Washington DC, 2013.

[33] Clark DA. \& Beck AT.: Cognitive Therapy for Anxiety Disorders, The Guilford Press, New York, 2010. 